\title{
Heinz Bolsenkötter
}

\section{Modernisierung des Bilanzrechts - Auswirkungen auf öffentliche Unternehmen und Verwaltungen}

Anstaltsrecht; Bilanzrechtsmodernisierung; Eigenbetriebsrecht; Gemeindehaushaltsrecht; Handelsrecht; Haushaltsreform; Internationalisierung der Rechnungslegung; juristische Problematik von Gesetzesverweisen.

Das Bilanzrechtsmodernisierungsgesetz (BilMoG; s. auch ZögU 2008, S. 302 ff.) hat inzwischen den Bundestag und den Bundesrat passiert und ist verkündet worden und in Kraft getreten. ${ }^{1}$ Schwerpunktmäßig ändert es die Rechnungslegungsvorschriften des Handelsgesetzbuchs (HGB) und des HGB-Einführungsgesetzes, bringt aber als Artikelgesetz auch zahlreiche damit korrespondierende Änderungen in anderen Gesetzen.

Die grundlegenden Ziele und Ansatzpunkte - insbesondere eine moderate Annäherung an internationale Rechnungslegungsstandards mit Stärkung der Informationsfunktion der Rechnungslegung unter Beibehaltung des Systems der Grundsätze ordnungsmäßiger Buchführung und der Systematik des HGB - wurden beibehalten; damit wird auch den von den Neuregelungen Betroffenen großenteils der unmittelbare Umgang mit den komplexen und umfänglichen internationalen Standards erspart.

Allerdings haben die Diskussionen in der Schlussphase der Gesetzgebung sowie die weltweite Finanz- und Wirtschaftskrise zu einigen wesentlichen sachlichen Änderungen der ursprünglichen Intentionen geführt, wobei tendenziell mehr Zurückhaltung hinsichtlich von Bilanzansatz- und Bewertungsmöglichkeiten die Gesetzgebung geprägt hat. Es seien genannt

- Beschränkung der Zeitwertbewertung von Finanzinstrumenten des Handelsbestands auf Kreditinstitute ( $\$ 340$ e Abs. 3 und $4 \mathrm{HGB}^{2}$ ); die Absicht, diese auch den Unternehmen der Realwirtschaft zu gestatten, wurde also aufgegeben. Kreditinstitute müssen die Zeitwertbewertung mit der Bildung eines Sonderpostens verbinden, der sowohl als Risikopufferung wie auch als Ausschüttungssperre wirkt.

- Dennoch ist der Zeitwert als Bewertungsmaßstab (wie im Regierungsentwurf vorgesehen) ausdrücklich in das Gesetz aufgenommen worden (§ 255 Abs. 4 HGB); er hat Bedeutung bei der Bildung von Bewertungseinheiten ( $\$ 254$ HGB) und bei der

1 Gesetz zur Modernisierung des Bilanzrechts (Bilanzrechtsmodernisierungsgesetz BilMoG vom 25.05.2009, BGBl. I vom 28.05.2009, S. 1102).

2 Gesetzesverweise ohne weitere Angaben beziehen sich in diesem Beitrag auf die aktuelle Version in der Fassung des BilMoG. 
Verrechnung von Vermögensgegenständen mit Altersversorgungsverpflichtungen ( $\S 246$ Abs. 2, 253 Abs. 1 S. 3 und 4 HGB).

- Die vorgesehene Pflicht zur Aktivierung selbst geschaffener immaterieller Gegenstände des Anlagevermögens wurde durch ein Wahlrecht ersetzt (§ 248 Abs. 2 S. 1 HGB); für Marken, Drucktitel, Verlagsrechte, Kundenlisten oder vergleichbare immaterielle Gegenstände des Anlagevermögens gilt ein Aktivierungsverbot.

- Auf die Einführung einer Aktivierungspflicht für aktive latente Steuern wird verzichtet; es bleibt also beim Aktivierungswahlrecht (§ 274 Abs. 1 HGB).

- Für die Festlegung des Konsolidierungskreises wird - wie von der Regierung vorgeschlagen - das Beteiligungskriterium aufgegeben; es wird aber auch nicht mehr auf die einheitliche Leitung, sondern auf das Konzept des beherrschenden Einflusses in Verbindung mit einer Chancen-Risiko-Betrachtung abgestellt ( 290 Abs. 1 und 2 HGB). Dies soll zu einer Einbeziehung von Zweckgesellschaften in den HGB-Konzernabschluss zwingen.

Im Übrigen wurden im Wesentlichen die Ansatzpunkte und Ziele, die bereits den Regierungsentwurf geprägt hatten, weiter verfolgt und gesetzgeberisch umgesetzt. Über die im vorjährigen ZögU-Beitrag (ZögU 2008 S. 306 ff.) aufgeworfenen Fragen hinsichtlich der Auswirkungen im öffentlichen Sektor ist es mittlerweile zu einer - im Wesentlichen zustimmenden - Diskussion gekommen. Die bereits gegebenen Hinweise seien also im Folgenden fortgeführt, aktualisiert und ergänzt.

\section{Ansatzpunkte und Ziele des BilMoG, Inkrafttreten und Übergangsvorschriften}

Die Ansatzpunkte und Ziele des BilMoG - moderate Annäherung an die International Financial Reporting Standards (IFRS) bei Aufrechthaltung des Systems der Grundsätze ordnungsmäßiger Buchführung, der Maßgeblichkeit der Handelsbilanz für die Steuerbilanz, der Funktion der HGB-Bilanz für die Ausschüttungsbemessung - wurden beibehalten und wohl auch wie angestrebt gesetzgeberisch realisiert. $\mathrm{Zu}$ begrüßen ist es auch, dass damit eine im Vergleich zu den umfangreichen und wenig übersichtlichen IFRS „kostengünstigere und einfachere Alternative" erfolgreich gesetzgeberisch umgesetzt wurde, die auch von kleinen und mittleren Unternehmen verarbeitet werden kann.

Die oben einleitend angesprochenen sachlichen Änderungen im Laufe des Gesetzgebungsverfahrens - die durchaus gerechtfertigt sind - haben ein Ziel der Reformen (die Annäherung an die IFRS) etwas zurückdrängt. So gehört es zu den Grundanliegen der IFRS, Bilanzansatz- und Bewertungswahlrechte möglichst zu vermeiden; neben der Beseitigung solcher Wahlrechte ist es aber nun doch wieder zu deren Zulassung in einigen Fällen gekommen. Auch ist die Bewertung zum beizulegenden Zeitwert (fair value) nicht in dem Maße in das HGB übernommen worden wie das ursprünglich beabsichtigt war; 
dies beruht darauf, dass nach vorherrschender Meinung in Deutschland ${ }^{3}$ weltweit die Zeitwertbewertung von zum Handel bestimmten Finanzinstrumenten zur Verschärfung der aktuellen Finanzkrise beigetragen hat. Bei Kreditinstituten soll der erwähnte korrespondierende obligatorische Sonderposten antizyklisch wirken. ${ }^{4}$

Im Wesentlichen unverändert gegenüber dem Regierungsentwurf sind durch die Reform kleinere Einzelkaufleute von der Pflicht zur Buchführung und zur Erstellung eines Inventars befreit ( $\S 241 \mathrm{a}, 242$ Abs. 4 HGB) und wurden die Schwellenwerte der $\S \S 267$ (Definition der kleinen und mittelgroßen Kapitalgesellschaften) und 293 HGB (größenabhängige Befreiungen von der Pflicht, einen Konzernabschluss und einen Konzernlagebericht aufzustellen) angehoben.

Das Gesetz trat (Art. 15 BilMoG) am Tag nach seiner Verkündung, also am 29.05.2009, in Kraft. Die Übergangsvorschriften (Art. 66 EGHGB) sehen zusammengefasst Folgendes für die erstmalige Anwendung vor:

- $\quad$ Erleichternde und befreiende Vorschriften: ab 01.01.2008

- $\quad$ Erweiterte Berichterstattungspflichten: ab 01.01.2009

- Handelsbilanzielle wesentliche Änderungen: ab 01.01.2009 optional, ab 01.01.2010 obligatorisch

- $\quad$ Regelung zu selbst erstellten immateriellen Gegenständen des Anlagevermögens: ab 01.01 .2010

Besondere, z. T. langfristig wirkende Übergangsregelungen bestehen hinsichtlich geänderter Bilanzierungs- und Bewertungsvorschriften, soweit schon bisher bestehende Bilanzposten davon betroffen sind (Art. $67 \mathrm{EGHGB})^{5}$.

\section{Wesentliche Reformmaßnahmen}

Wesentliche Änderungen im Bereich der Rechnungslegung betreffen:

\section{Allgemeines}

- Der Grundsatz der wirtschaftlichen Betrachtungsweise bei der Zurechnung von Vermögensgegenständen in der Bilanz hat Vorrang vor dem Kriterium des juristischen Eigentums ( $§ 246$ Abs. 1 HGB).

- $\quad$ Die Grundsätze der Bilanzansatzstetigkeit (§ 246 Abs. 3 HGB) und der Bewertungsstetigkeit (§ 252 Abs. 1 Nr. 6 HGB) sind jetzt zwingend (als Muss-Vorschriften) vorgegeben.

- $\quad$ Das Verrechnungsverbot des $\S 246$ Abs. 2 HGB wird in einigen Fällen durch einen Zwang zum Nettoausweis modifiziert:

- Verrechnungsgebot für dem Zugriff der übrigen Gläubiger entzogene Vermögensgegenstände, die ausschließlich der Erfüllung von Altersversorgungsver-

3 Dies betrifft die öffentliche ebenso wie die Fachdiskussion; s. u. a. Bieg u. a., Die Saarbrücker Initiative gegen den Fair Value (2008), sowie anschließende Diskussion.

4 Ernst/Seidler (2009), S. 769.

5 Zu Einzelheiten s.: Institut für Wirtschaftsprüfer in Deutschland e. V. (2009a). 
pflichtungen dienen, ebenso auch für Aufwendungen und Erträge aus der Abzinsung und aus dem zu verrechnenden Vermögen; ein Aktivsaldo ist gesondert auszuweisen.

- $\quad$ Für nicht eingeforderte ausstehende Einlagen auf das eingeforderte Kapital ( 272 Abs. $1 \mathrm{HGB}$ ) sowie für eigene Anteile ist die offene Absetzung vom gezeichneten Kapital (§ 272 Abs. 1a HGB) vorgeschrieben, im letzteren Falle unter Verrechnung von Differenzen aus den Anschaffungskosten mit frei verfügbaren Rücklagen.

- $\quad$ Für latente Steuern besteht ein Wahlrecht zwischen Brutto- und Nettoausweis (§ 274 Abs. 1 HGB; s. auch weiter unten).

- Zur Aufrechnung von Chancen und Risiken kommt es bei der Bildung von Bewertungseinheiten aus Sicherungsgeschäften (§ 254 HGB; handelsrechtlich bisher nicht geregelt, allerdings enthielt bereits das Steuerrecht in $\S 5$ Abs. 1a EStG eine Regelung hierzu).

- $\quad$ Der Grundsatz der sogenannten umgekehrten Maßgeblichkeit (§ 5 Abs. 1 S. 2 EStG a. F.) wird aufgehoben und durch besondere Nachweispflichten ersetzt ( 55 Abs. 1 S. 2 und $3 \mathrm{EStG}$ ); in diesem Zusammenhang entfallen auch damit korrespondierende bisherige HGB-Regelungen ( $\$ 254 \mathrm{HGB}$ a. F. über steuerrechtliche Abschreibungen, § 273 HGB a.F. über Sonderposten mit Rücklageanteil, §§ 279 Abs. 2 bis 281 HGB a. F. über steuerrechtliche Vorschriften einschließlich solcher zum Wertaufholungsgebot). Nach bisherigem Recht gebildete Sonderposten können beibehalten werden oder sind unmittelbar in die Gewinnrücklagen einzustellen (Art. 67 Abs. 3 EGHGB).

\section{Bilanzansatz}

- Der Geschäfts- oder Firmenwert gilt als zeitlich begrenzt nutzbarer Vermögensgegenstand (§ 246 Abs. 1 S. 3 HGB); ist er derivativ, besteht Aktivierungspflicht, sonst (wie bisher) Aktivierungsverbot.

- Für selbst geschaffene immaterielle Vermögensgegenstände des Anlagevermögens wurde jetzt nach einigen Diskussionen ein Ansatzwahlrecht (mit sachlicher Begrenzung) kodifiziert, wobei nach $\S 268$ Abs. 8 S. 1 HGB eine korrespondierende Ausschüttungssperre greift. Aktivierbar sind nur die Kosten der Entwicklungsphase (unter Ausschluss derjenigen der Forschungsphase; § 255 Abs. 2a HGB).

- Die bisherige Bilanzierungshilfe für Ingangsetzungs- und Erweiterungsaufwendungen (§ 269 HGB a.F.) wird aufgehoben; das Vollständigkeitsgebot des § 246 Abs. 2 HGB umfasst Bilanzierungshilfen nicht. Bisher aktivierte Bilanzierungshilfen können beibehalten werden (Art. 67 Abs. 5 EGHGB).

- Vorschriften zur Bildung von Aufwandsrückstellungen für unterlassene Instandhaltung u. ä. (weitgehende Wahlrechte; $§ 249$ Abs. 1 S. 3, Abs. 2 HGB a.F.) wurden aufgehoben (bestehen bleiben die Passivierungspflichten nach $\S 249$ Abs. 1 S. 2 HGB für innerhalb von drei Monaten nachgeholte Instandhaltungsaufwendungen, für im Folgejahr nachgeholte Aufwendungen für Abraumbeseitigung und für Ku- 
lanzleistungen); bestehende Rückstellungen - soweit nicht im letzten vor dem 01.01.2010 beginnenden Geschäftjahr gebildet - können beibehalten oder in Gewinnrücklagen eingestellt werden (Art. 67 Abs. 3 EGHGB).

- Für latente Steuern gibt es nach der abschließenden gesetzgeberischen Lösung mehrere Wahlrechte ( $\$ 274$ Abs. 1 HGB $)^{6}$. Zulässig ist eine Gesamtdifferenzbetrachtung mit Passivierungspflicht für einen Passivsaldo und Aktivierungswahlrecht für einen Aktivsaldo. Die sich ergebende Steuerbelastung und die sich ergebende Steuerentlastung können aber auch unverrechnet angesetzt werden; steuerliche Verlustvorträge sind bei der Berechnung aktiver latenter Steuern für die nächsten fünf Jahre zu berücksichtigen ${ }^{7}$. Konzeptionell wird (wie auch bereits nach dem Regierungsentwurf) von der GuV-orientierten auf die bilanzorientierte Sicht umgestellt.

\section{Bewertung}

- Bei den Herstellungskosten ( $\$ 255$ Abs. 2 S. 2 HGB) wird die Bewertungsuntergrenze an die steuerliche Bewertungsuntergrenze angeglichen (Einschränkung von Bewertungswahlrechten). Die Einbeziehungswahlrechte für Verwaltungskosten, Kosten für soziale Einrichtungen, freiwillige soziale Leistungen und für die betriebliche Altersversorgung sowie für fertigungsbezogene Fremdkapitalzinsen bleiben bestehen.

- Aufgehoben werden die Wahlrechte für Abschreibungen wegen künftiger Wertschwankungen sowie für Abschreibungen im Rahmen vernünftiger kaufmännischer Beurteilung (§ 253 Abs. 3 S. 3, Abs. 4 HGB); die Möglichkeit der außerplanmäßigen Abschreibung bei nur vorübergehender Wertminderung wird auf Finanzanlagen beschränkt ( $\$ 253$ Abs. 3 S. 4 HGB) und die Wertaufholung wird nun generell vorgeschrieben (§ 253 Abs. 5 S. 1 HGB).

- Verbindlichkeiten sind zu ihrem Erfüllungs- (bisher Rückzahlungs-)betrag anzusetzen (§ 253 Abs. 1 S. 2 HGB). Das hat einerseits klarstellende Bedeutung, weil es sprachlich umfassender ist; es besagt andererseits aber auch, dass - in Durchbrechung des Stichtagsprinzips - künftige Preis- und Kostensteigerungen zu berücksichtigen sind.

- Markante Änderungen ergeben sich bei den Rückstellungen:

- Rückstellungen mit einer Restlaufzeit von mehr als einem Jahr sind mit dem ihrer Restlaufzeit entsprechenden durchschnittlichen Marktzinssatz der vergangenen sieben Geschäftsjahre abzuzinsen (§ 253 Abs. 2 S. 1 HGB).

$6 \quad$ Zu Einzelheiten s.: Institut der Wirtschaftsprüfer in Deutschland e. V. (2009b).

7 Bei diesen Wahlmöglichkeiten für den Ausweis darf aber nicht übersehen werden, dass geforderte Anhangangaben stets zu exakten Ermittlungen und Berechnungen zwingen. Nach $\$ 285$ Nr. 29 HGB ist im Anhang anzugeben, ,auf welchen Differenzen oder steuerlichen Verlustvorträgen die latenten Steuern beruhen und mit welchen Steuersätzen die Bewertung erfolgt ist". Der Rechtsausschuss des Bundestages hält diese Angabe selbst dann für erforderlich, wenn in der Bilanz latente Steuern - etwa aufgrund der Gesamtdifferenzbetrachtung - nicht ausgewiesen sind (BT-Drs. 16/12407 S. 116). 
- Altersversorgungsverpflichtungen ${ }^{8}$, vergleichbare langfristige Verpflichtungen und Rentenverpflichtungen, für die eine Gegenleistung nicht mehr zu erwarten ist, dürfen pauschal mit dem durchschnittlichen Marktzinssatz abgezinst werden, der sich bei einer angenommenen Restlaufzeit von 15 Jahren ergibt (§ 253 Abs. 2 S. 2 u. 3 HGB).

Die Diskontierungssätze werden aufgrund einer noch zu erlassenden Rechtsverordnung von der Bundesbank verbindlich vorgegeben.

Anmerkung: Das Steuerrecht folgt diesen Änderungen nicht. Es bleibt also bei der Abzinsung von Rückstellungen mit 5,5\% p. a. (§ 6 Abs. 1 Nr. 3a Buchstabe e EStG), beim Stichtagsprinzip ( $(6$ Abs. 1 Nr. 3a Buchstabe f EStG) und bei der Bewertung von Pensionsrückstellungen zum steuerlichen Teilwert (§ 6a EStG).

- Altersversorgungsverpflichtungen, deren Höhe sich nach dem Zeitwert bestimmter Wertpapiere richtet, sind mit diesem Zeitwert anzusetzen $(\S 253$ Abs. 1 S. 3 HGB); sind diese Vermögensgegenstände dem Zugriff der übrigen Gläubiger entzogen, sind sie (wie bereits erwähnt) mit den Schulden zu verrechnen, wobei sich auch ein - dann gesondert auszuweisender - Aktivsaldo ergeben kann ( $\S 253$ Abs. 1 S. 4, 246 Abs. 2 S. 2 u. 3 HGB).

- Für die Umrechnung von auf fremde Währung lautenden Vermögensgegenständen und Verbindlichkeiten besteht nunmehr erstmals eine allgemeine Regelung ( $256 \mathrm{a}$ HGB). Es gilt der Devisenkassamittelkurs am Abschlussstichtag, wobei bei einer Restlaufzeit von einem Jahr oder weniger keine Beschränkung auf die Anschaffungskosten als Obergrenze eingreift.

- $\quad$ Von den Bewertungsvereinfachungsverfahren des $\S 256$ HGB sind jetzt nur noch die FIFO- und die LIFO-Methode zulässig.

- $\quad$ Eine Ausschüttungssperre ( $\$ 268$ Abs. 8 HGB) gilt in Höhe

- $\quad$ aktivierter selbst erstellter immaterieller Vermögensgegenstände des Anlagevermögens,

- von in der Bilanz ausgewiesenen aktiven latenten Steuern,

- $\quad$ eines Aktivsaldos zeitwertbewerteter Vermögensgegenstände für die Altersversorgung abzüglich hierfür passivierter latenter Steuern,

soweit nicht noch frei verfügbare Rücklagen unter Berücksichtigung eines Ergebnisvortrags verbleiben.

- $\quad$ Die Wirkung einer Ausschüttungssperre hat bei Kreditinstituten und Finanzdienstleistungsinstituten auch der Sonderposten nach $\S 340$ e Abs. 4 HGB aus der Zeitwertbewertung von Finanzinstrumenten des Handelsbestandes.

\section{Konzernrechnungslegung}

- Das Beteiligungskriterium des $\S 290$ Abs. 1 S. 1 HGB a.F. für den Begriff des Tochterunternehmens wird gestrichen; entscheidend ist künftig nur noch die Möglichkeit, einen beherrschenden Einfluss auszuüben (der Regierungsentwurf hatte

8 Die Ansatzwahlrechte des Art. 28 Abs. 1 EGHGB für Altzusagen und für mittelbare Verpflichtungen bleiben unverändert bestehen. 
noch die einheitliche Leitung vorgesehen). Der beherrschende Einfluss definiert sich (§ 290 Abs. 2 HGB) über die Mehrheit der Gesellschafterstimmrechte, das Recht eines Gesellschafters zur Bestellung der Mehrheit der Entscheidungsträger, die Bestimmung der Geschäftspolitik durch Beherrschungsvertrag oder Satzungsbeschluss oder das Tragen der Mehrheit der Chancen und Risiken eines zweckbestimmten Unternehmens. Diese Regelungen sind maßgeblich von Erfahrungen aus der jüngsten Finanzkrise mit beeinflusst worden.

- Für die auf den Konzernabschluss angewandten Konsolidierungsmethoden gilt nun der Grundsatz unbedingter Stetigkeit (§ 297 Abs. 3 S. 2 HGB; bisher ,sollen beibehalten werden").

- Die Kapitalkonsolidierung ist künftig nur noch nach der Neubewertungsmethode durchzuführen (§ 301 Abs. 1 S. 2 HGB); die Buchwertmethode ist nicht mehr zulässig. Rückstellungen für latente Steuern sind dabei nach den Vorschriften für den Jahresabschluss zu bewerten ( $\$ 301$ Abs. 1 S. 3 HGB).

- Hingegen ist für die Behandlung assoziierter Unternehmen (,,at equity“) künftig nur noch die Buchwertmethode zugelassen (§ 312 HGB).

- $\quad$ Die bisher schon bedeutungslose Regelung zur Kapitalkonsolidierung bei Interessenzusammenführung ( $\$ 302 \mathrm{HGB}$ ) wurde gestrichen.

- $\quad$ Die Regelung für latente Steuern entspricht im Wesentlichen der für den Jahresabschluss (§ 306 HGB).

- Neu ist die Regelung zur Umrechnung von auf fremde Währung lautenden $A b$ schlüssen (§ 308a HGB). Für die Posten der Bilanz gilt der Devisenkassamittelkurs zum Abschlussstichtag; das Eigenkapital ist zum historischen Kurs umzurechnen. Die Posten der GuV-Rechnung sind zum Durchschnittskurs umzurechnen. Umrechnungsdifferenzen sind beim Konzerneigenkapital nach den Rücklagen in einem gesonderten Posten zu verrechnen.

\section{Berichterstattungspflichten}

- In Verbindung mit der Novellierung von Rechnungslegungsvorschriften wurden zahlreiche Regelungen zu Angabepflichten im Anhang/Konzernanhang (§§ 285, 314 HGB) neu eingeführt oder ergänzt bzw. differenziert; eine Einzeldarstellung erscheint uns hier entbehrlich.

- Eine Kapitalflussrechnung und einen Eigenkapitalspiegel müssen künftig auch solche kapitalmarktorientierte Kapitalgesellschaften (§ 264d HGB) als Bestandteil ihres Jahresabschlusses erstellen, die nicht zur Aufstellung eines Konzernabschlusses verpflichtet sind ( $\$ 264$ Abs. 1 S. 2 HGB). Eine Segmentberichterstattung ist optional; allerdings können andere Vorschriften zur Segmentierung im Rechnungswesen zwingen, etwa $\S 10$ Abs. 3 EnWG für zu entflechtende Tätigkeiten und $\S 8$ Abs. 9 KStG hinsichtlich der steuerrelevanten Spartenabgrenzung.

- Diese Gesellschaften ( $\$ 264 \mathrm{~d}$ HGB) haben ferner im Lagebericht ihr internes Kontrollsystem und ihr Risikomanagementsystem im Hinblick auf die Rechnungslegung zu beschreiben ( $§ 289$ Abs. 5 HGB). 


\section{Auswirkungen im öffentlichen Sektor}

\section{Allgemeines}

Die angesprochenen, von der HGB-Reform betroffenen Vorschriften gelten teils für alle Kaufleute (Erster Abschnitt des Dritten Buchs des HGB; $\S \S 238$ - 263), teils für Kapitalgesellschaften und bestimmte Personenhandelsgesellschaften (Zweiter Abschnitt des Dritten Buchs des HGB; $\S \S 264$ - 335), soweit nicht ihr Anwendungsbereich im Einzelnen noch weiter eingegrenzt ist. Im öffentlichen Sektor wirken die Neuregelungen nur dann unmittelbar, wenn eine Betätigung der öffentlichen Hand die Kaufmannseigenschaft hat (was nicht eigene Rechtspersönlichkeit voraussetzt) oder wenn sie in einer Rechtsform stattfindet, die vom HGB erfasst wird; im Übrigen können ggf. Verweisregelungen zum Tragen kommen, wobei sich die Problematik ergibt, ob solche Verweise dyamisch zu interpretieren sind, also auch eine spätere Änderung der über den Verweis anzuwendenden Vorschrift (hier also im HGB) mit erfassen sollen. Die Verweise sind insofern z. T. eindeutig (in der Regel dann statisch), oft aber auch sprachlich allgemein gehalten und dann also interpretationsbedürfig. ${ }^{9}$

Die in dem ZögU-Beitrag 2008 (S. 306 ff.) angesprochene Problematik hat sich also in der Zwischenzeit grundsätzlich nicht geändert; lediglich inhaltlich führt jetzt die abschließende BilMoG-Fassung zu einigen neuen Aspekten im Hinblick auf den von uns gesehenen Anpassungsbedarf bei öffentlichen (insbesondere kommunalrechtlichen) Rechnungslegungsvorschriften, um die Rechnungslegung sowohl innerhalb des öffentlichen Sektors als auch im Vergleich zum unternehmerischen Bereich nicht zu stark auseinander driften zu lassen. Eine aktuelle Literaturdiskussion hat die in der ZögU von uns geäußerten Besorgnisse im Wesentlichen bestätigt. Wir geben folgende Hinweise:

Vogelpoth/Poullie/Vo $\beta^{10}$ : Diese Autoren gehen den sachlichen und rechtlichen Problemen (einschließlich der Verweisproblematik) nach, die sich aus den inhaltlichen Unterschieden der Regelungen und den Überlagerungen verschiedener Rechtssphären ergeben. Sie bestätigen mit detaillierter Analyse die Sicht, dass mit verständiger, sachbezogener Interpretation alleine die in den rechtlichen Grundlagen der Rechnungslegung entstandenen Diskrepanzen nicht überbrückt werden können.

Bauer/Kirchmann $/ \mathrm{Sa} \beta^{11}$ : Im Beitrag dieser Autoren werden vor dem Hintergrund der gegebenen Sach- und Rechtsproblematik einige Teilbereiche aufgegriffen, in denen die HGB-Reform zu einem Folgereformbedarf im Kommunalrecht führt, wenn denn Harmonisierung gleich oder ähnlich gelagerter Sachverhalte als ein übergeordnetes Ziel der Rechtsordnung angesehen wird.

9 Aktuell hierzu: Vogelpoth/Poullie/Voß (2009)

10 Norbert Vogelpoth, Michael Poullie, Katharina Voß, Probleme im Zusammenwirken von HGB, BilMoG und kommunaler Rechnungslegung, in: Die Wirtschaftsprüfung 2009 S. 83.

11 Ludwig Bauer, Ulrich Kirchmann, Johannes Saß: Mögliche Auswirkungen durch das Bilanzrechtsmodernisierungsgesetz (BilMoG) auf die kommunale Doppik, in: Die Wirtschaftsprüfung 2009, S. 143. 
Ellerich $^{12}$ : Er sieht selbstverständlich ebenfalls sowohl die bereits vor der HGBNovellierung bestehenden Unterschiede in der Ländergesetzgebung ${ }^{13}$ als auch die künftigen Konsequenzen aus den HGB-Änderungen; einen Aspekt betont er besonders: Soweit im Gemeindehaushaltsrecht auf die Grundsätze ordnungsmäßiger Buchführung (GoB) verwiesen wird, greifen einschlägige HGB-Änderungen mit GoB-Wirkung auch auf die Gemeindehaushalte durch, soweit dem nicht ausdrückliche kommunalrechtliche Regelungen entgegenstehen ${ }^{14}$. Als wesentliches Beispiel für die GoB-Fortentwicklung nennt Ellerich die neuen Bewertungsregeln für Rückstellungen. Allerdings kann man derartige punktuelle Einwirkungen des BilMoG auch nicht gerade als glücklich empfinden, wenn im Übrigen ein früherer Stand des HGB als Referenzmodell das reformierte kommunale Haushaltsrecht geprägt hat.

\section{Einzelhinweise}

\section{a) Kapitalgesellschaften}

Kapitalgesellschaften und (bestimmte ${ }^{15}$ ) Personalhandelsgesellschaften sind stets Kaufleute und haben darüber hinaus die besonderen Vorschriften des Zweiten Abschnitts des Dritten Buchs des HGB zu beachten; die öffentliche Trägerschaft ändert insofern nichts an der Geltung der HGB-Vorschriften in der jeweils gültigen, künftig also in der durch das BilMoG geänderten Fassung. Das Kommunalrecht verpflichtet die Kommunen, bei ihren Beteiligungsgesellschaften sicherzustellen, dass in jedem Falle die strengeren Vorschriften für große Kapitalgesellschaften angewendet werden. Kapitalmarktorientierte Kapitalgesellschaften ( $(264 \mathrm{~d}$ HGB) gelten stets als große (§ 267 Abs. 3 S. 2 HGB); bei Kommunen dürfte diese Regelung kaum relevant werden.

\section{b) Eigenbetriebe ohne Rechtspersönlichkeit und rechtsfähige Anstalten}

Diese Rechtsformen haben vor allem bei Kommunen erhebliche praktische Bedeutung, und zwar primär für sog. wirtschaftliche Bestätigungen im Sinne des Kommunalrechts, aber auch vielfach für nicht wirtschaftliche (in der Regel hoheitliche) Betätigungen, die kommunalrechtlich ebenfalls definiert sind. Das Eigenbetriebsrecht enthält Rechnungslegungsvorschriften, die weitgehend dem HGB-Maßstab für große Kapitalgesellschaften

12 Marian Ellerich, Keine Auswirkungen des BilMoG auf den kommunalen Jahresabschluss? - Zugleich ein Plädoyer gegen allzu bunte Blumensträuße, Editorial in: Die Wirtschaftsprüfung Heft 7/2009.

13 Aktuell hierzu: PricewaterhouseCoopers (2009); Marettek/Hellenbrand/Detemple (2009); Mühlenkamp/ Glöckner (2009).

$14 \mathrm{Ob}$ sich juristisch noch ein weiterer Problemaspekt ergibt, wenn das Kommunalrecht nicht auf die GoB, sondern auf die „Grundsätze ordnungsmäßiger Buchführung von Gemeinden“ verweist (so Rheinland-Pfalz und das Saarland, ähnlich Bayern), mag hier nur als Frage aufgeworfen werden.

15 Im Zweiten Abschnitt des Dritten Buchs des HGB den Kapitalgesellschaften gleichgestellt. 
entsprechen. Das kommunale Anstaltsrecht verweist hinsichtlich der Rechnungslegung regelmäßig auf das Eigenbetriebsrecht, ${ }^{16}$ sodass insofern hier nicht weiter differenziert werden muss. Hingegen hat die Frage nach der Kaufmannseigenschaft konkrete Bedeutung, da hiervon die unmittelbare Geltung der HGB-Vorschriften des Ersten Abschnitts des Dritten Buchs des HGB abhängt; im Regelfall wird dies zusammenfallen mit dem Vorliegen einer wirtschaftlichen Betätigung im Sinne des Kommunalrechts. Für nichtwirtschaftliche Betätigungen wird übrigens gelegentlich auch die Form einer sog. eigenbetriebsähnlichen Einrichtung gewählt, bei der das jeweilige Eigenbetriebsrecht nicht in voller Strenge angewendet wird.

Von besonderem Interesse sind hier die Betriebe/Anstalten mit Kaufmannseigenschaft, für die also ein Teil der BilMoG-Regelungen unmittelbar gilt und für die im Übrigen Sonderregelungen und HGB-Verweise nebeneinander greifen. ${ }^{17}$ Zwei Verweise sind hier vor allem relevant: die auf die HGB-Vorschriften zur Buchführung, zum Inventar und zur Aufbewahrung (z. B. § 19 Abs. 2 EigVO NRW) sowie die auf ergänzende Vorschriften zum Jahresabschluss (z. B. § 21 EigVO NRW). In beiden Fällen lassen es die Verweise nach ihrer Formulierung offen, ob sie statisch oder dynamisch gemeint sind; es spricht viel dafür, dass sie einer gesetzestechnischen Vereinfachung dienen und dass mithin eine dynamische Interpretation gerechtfertigt ist. ${ }^{18}$ Insbesondere hinsichtlich der aktuellen Neuregelungen zum Jahresabschluss (allgemeine Vorschriften, Ansatz- und Bewertungssowie sonstige Vorschriften) mögen hier aber auch gewisse rechtliche Bedenken verbleiben, die sich aus dem Ausmaß und dem Gewicht der vom BilMoG gebrachten HGBÄnderungen ergeben; sie werden immerhin häufig als die wesentlichsten seit dem Bilanzrichtliniengesetz charakterisiert. Sachlich dürften aber kaum Zweifel daran bestehen, dass eine dynamische Interpretation, also die faktische Übernahme der BilMoG-Reform in das Eigenbetriebsrecht, sinnvoll ist. ${ }^{19}$

Eine kommentierende Anmerkung zum Regelungsgehalt des BilMoG in Bezug auf die Eigenbetriebe/Anstalten mag hier noch gerechtfertigt sein. Die Sonderregelungen des Eigenbetriebsrechts zur Rechnungslegung sind nicht so umfänglich, dass eine integrierende Übernahme der neuen HGB-Vorschriften nennenswerte Schwierigkeiten oder gar systematische Konflikte mit sich bringen würde. Für eine konsequente Anpassung der Rechnungslegung der Eigenbetriebe/Anstalten an die der Kapitalgesellschaften dürfte es also nennenswerte Hindernisse nicht geben. Das schließt es natürlich nicht aus, dass

16 Bolsenkötter/Dau/Zuschlag, Rn. A 53 ff., S. 622 f.

17 Hierzu nach bisherigem Recht: Bolsenkötter/Dau/Zuschlag, Rn. W 280 ff., S. 348 ff.

18 Bolsenkötter/Dau/Zuschlag, Rn. W 789 ff., S. 584 f.; Vogelpoth/Poullie/Voß, S. 85.

19 In diesem Zusammenhang mag es auch interessant sein, dass das Land Brandenburg in der Schlussphase der gesetzgeberischen Arbeiten am BilMoG eine Neufassung seiner Eigenbetriebsverordnung beschlossen und verkündet hat (Eigenbetriebsverordnung - EigV - vom 26. März 2009, GVB1. Brandenburg, Teil II, vom 27. April 2009, S. 150). Die HGB-Verweise ( $\S 19$ Abs. 2 und 21 Abs. 1 S. 3 ) in dieser Verordnung entsprechen inhaltlich und in der Diktion den auch sonst im Eigenbetriebsrecht üblichen Verweisen auf die HGBRechnungslegung. Dies dürfte ein weiteres Indiz für die Rechtfertigung einer dynamischen Interpretation der Verweise im Eigenbetriebsrecht sein, da ja dem Verordnungsgeber die unmittelbar vor ihrem Abschluss stehende Arbeit auf Bundesebene am BilMoG bekannt war. Auch im Freistaat Sachsen wurde etwa zeitgleich an Änderungen des Eigenbetriebsrechts gearbeitet. 
legislatorische Klarstellungen und Detailregelungen zu einzelnen Sachverhalten teils sinnvoll, teils geboten erscheinen.

\section{c) Kernverwaltungen der Kommunen}

Obwohl sich die länderrechtlichen Vorschriften zum reformierten Haushaltsrecht - soweit sie obligatorisch oder optional ein doppisches, auf dem Ressourcenverbrauchskonzept basierendes System vorsehen - am Referenzmodell der HGB-Rechnungslegung orientieren, wurde gesetzestechnisch bei der Umsetzung ein deutlich anderer Weg beschritten als im kommunalen Eigenbetriebs-/Anstaltsrecht. Die Regelungen zur Rechnungslegung wurden weitestgehend haushaltsrechtlich ausformuliert - also nicht durch die Technik der HGB-Verweise übernommen - und dabei z. T. auch abweichend vom HGB-Vorbild gestaltet; für die Eröffnungsbilanzen bestehen regelmäßig einige besondere Vorschriften. Die wenigen HGB-Verweise auf Einzelvorschriften sind in der Regel ausdrücklich statisch formuliert. Die bereits erwähnten Verweise auf die Grundsätze ordnungsmäßiger Buchführung sind wohl zulässigerweise dynamich zu interpretieren $^{20}$, da die GoB generell als ein in Entwicklung befindliches System anzusehen sind; BilMoG-Regeln können auf diesem Weg für die Haushalte aber selbstverständlich nur wirksam werden, soweit sie nicht mit haushaltsrechtlichen Vorgaben in Konflikt geraten.

Die Problematik, die sich aus der HGB-Novellierung durch das BilMoG ergibt, ist also im Bereich der Haushalte der Kernverwaltungen sowohl sachlich komplexer als auch legislatorisch etwas schwieriger zu lösen als im Eigenbetriebs-/Anstaltsrecht. Dies lässt natürlich andererseits eine baldige Beschäftigung der Landesgesetzgeber mit dieser Thematik umso dringlicher erscheinen.

Zwei Aspekte sind noch anzusprechen:

- Die Reformprozesse in den einzelnen Bundesländern befinden sich in recht unterschiedlichen Stadien. Z. T. bestehen noch mehrjährige Zeiträume/ Übergangsfristen für die Realisierung. Das mag Anlass für ein Überarbeiten der Reformen/ Reformpläne sein, um den betroffenen Gebietskörperschaften in möglichst vielen Fällen einen Reformeinstieg bereits auf einem BilMoG-konformen Stand zu ermöglichen.

- Die bisher legislatorisch umgesetzten Reformen enthalten im Ländervergleich - wie bereits erwähnt - etliche unterschiedliche Regelungen zu gleichen Sachverhalten. Die Annäherung haushaltsrechtlicher Regelungen an das BilMoG bietet also auch die Gelegenheit, insoweit mehr Konvergenz im Recht der Bundesländer zu erreichen.

20 Vogelpoth/Poullie/Voß, S. 86. Verweise auf die „GoB für Gemeinden“ können evtl. als einschränkend interpretiert werden. 


\section{d) Öffentliche Gesamtabschlüsse (Konzernabschlüsse)}

Ein Gesamtabschluss - bestehend aus Gesamtergebnisrechnung, Gesamtbilanz und Gesamtanhang -, ergänzt um einen Gesamtlagebericht und einen Beteiligungsbericht, kann als ein wesentliches Element der Haushaltsreformen angesehen werden. Reformgesetze sehen ihn generell vor, da er ein Gesamtbild von der Lage der Gebietskörperschaft unter Einschluss ausgegliederter Betätigungen vermittelt. Hinsichtlich der Konsolidierungstechniken wird üblicherweise auf die $\S \S 300$ bis 309 und 311, 312 HGB verwiesen, üblicherweise ausdrücklich statisch (Mecklenburg-Vorpommern, Nordrhein-Westfalen, Saarland, Sachsen, Thüringen). Die sich aus dem BilMoG ergebende Problematik geht jedoch weit über Fragen der Konsolidierungstechniken hinaus.

Die Einbeziehung von zwingend dem reformierten HGB unterliegenden Unternehmen und der zwingend dem nicht reformierten Haushaltsrecht unterliegenden Kernverwaltung führt zu einem grundlegenden Problem. Die Konzernrechnungslegung wird generell vom Grundsatz der einheitlichen Bilanzierung und Bewertung beherrscht, so selbstverständlich auch im öffentlichen Sektor; die HGB-Verweise erstrecken sich auch auf diesen wesentlichen Grundsatz. Zulässige bzw. hier sogar zwingende Unterschiede in den Jahresabschlüssen sind vor deren Zusammenführung in sog. Handelsbilanzen II auszugleichen, was hier zu einer partiellen Rücknahme der BilMoG-Reformen führen würde, ein weder sachlich noch prozedural befriedigendes Ergebnis. Erwähnt sei noch, dass öffentliche HGB-Konzerne - aus Sicht der Gebietskörperschaft Teilkonzerne -, also etwa Stadtwerke-Konzerne, selbstverständlich vollständig das HGB in der BilMoG-Fassung zu beachten haben.

Bei den anzuwendenden Konsolidierungstechniken dürften sich durch das BilMoG im Allgemeinen keine Probleme ergeben, allerdings mit einer wesentlichen Ausnahme. Das Haushaltsrecht mehrerer Bundesländer hat für die Kapitalkonsolidierung nur die sogenannte Buchwertmethode zugelassen, die 1t. HGB zulässige Neubewertungsmethode aber ausgeschlossen. Das BilMoG wählt in seinem neu gefassten § 301 Abs. 2 HGB genau den anderen Weg; hier ist die Buchwertmethode künftig nicht mehr zugelassen (was übrigens vielfachen Empfehlungen von Betriebswirten entspricht).

Bei den Gesamtabschlüssen kann es also zu einer Kumulierung von Problemen kommen; jedoch können die hier längeren Übergangsfristen der Länderreformgesetze zu entsprechenden legislatorischen Anpassungsmaßnahmen genutzt werden, was es der Praxis in vielen Fällen ersparen würde, mit diesen Schwierigkeiten zu kämpfen.

\section{Schlussbemerkungen}

Für die Landesgesetzgeber ist es gewiss keine glückliche Situation, dass in einer Phase, in der die seit Jahren diskutierten Haushaltsreformen legislatorisch z. T. realisiert, insgesamt aber bei Weitem noch nicht allgemein beschlossen und in der Praxis verständlicherweise in noch geringerem Maße umgesetzt sind, das für die doppische Lösung zu Grunde gelegte Referenzmodell der HGB-Rechnungslegung seinerseits 
Gegenstand einer Reform geworden ist. Der unterschiedliche Stand der Haushaltsreformen auf kommunaler Ebene und auf Länder-Ebene musste in diesem Beitrag nicht referiert werden; der Bund präferiert - soweit erkennbar - ein Modell der erweiterten Kameralistik. Die in diesem Beitrag gegebenen Hinweise auf Probleme hinsichtlich Berührungen, Überschneidungen und auch Konfliktsituationen im öffentlichen Sektor verkennen in keiner Weise, dass die nun beschlossene und verkündete Bilanzrechtsmodernisierung sinnvoll ist und dass sie auch zielgerecht und praxis-freundlich in die HGB-Systematik integriert wurde; gelegentliche Kritik am BilMoG ist - soweit ersichtlich - eher punktuell als grundsätzlich. Diese insgesamt positive Würdigung der HGBReform durch das BilMoG darf aber nicht den Blick dafür verstellen, dass den Haushaltsreformen ihr Referenzmodell nun partiell enteilt ist.

Die Auswirkungen im öffentlichen Sektor seien wie folgt zusammengefasst:

Kapitalgesellschaften und (bestimmte) Personalhandelsgesellschaften fallen unmittelbar und vollständig in den Geltungsbereich der Reform-Regelungen des BilMoG.

- (Sonstige) öffentliche Betätigungen mit Kaufmannseigenschaft unterliegen (mindestens) den für alle Kaufleute geltenden reformierten Vorschriften des Ersten Abschnitts des Dritten Buchs des HGB.

- Eigenbetriebe ohne Rechtspersönlichkeit und rechtsfähige Anstalten sind weitestgehend zu einer Rechnungslegung verpflichtet, die dem für große Kapitalgesellschaften geltenden HGB-Maßstab entspricht. Gesetzestechnisch wird dies z. T. über Sonderregelungen (z. B. Jahresabschluss-Formblätter), im Übrigen aber über Verweise auf das HGB erreicht. Diese Verweise sind allgemein nicht als statisch charakterisiert; eine - u. E. sinnvolle und praktisch unschwer umsetzbare wie auch juristisch vertretbare - dynamische Interpretation würde diese Einrichtungen zeitgleich in die HGB-Reformen einbinden. Ergänzende, der Anpassung dienende legislatorische Maßnahmen dürften sinnvoll und geboten, allerdings nicht sehr umfänglich sein.

- Im Gemeindehaushaltsrecht bedienen sich die Reformen der vergangenen Jahre weit-gehend autonom formulierter eigener Vorschriften und nur ergänzend der Verweis-technik, dies dann in der Regel ausdrücklich statisch, sodass die aktuellen BilMoG-Reformen nur sehr begrenzt ohne weitere Maßnahmen wirksam werden. Wenn denn institutionen-übergreifend eine möglichst einheitliche Rechnungslegung - zum leichteren Verständnis der Adressaten und zur Vergleichbarkeit der Informationen - als Ziel anerkannt wird, besteht hier ein Anpassungsbedarf, der ohne ein Tätig-werden des Gesetz-/Verordnungsgebers nicht gedeckt werden kann.

- Besonders gravierend erscheinen uns die Sachprobleme bei den öffentlichen Gesamtabschlüssen, weil hier Jahresabschlüsse von Unternehmen, sonstigen ausgegliederten Einheiten und der Kernverwaltung zusammengefasst werden, die nach der derzeitigen Rechtslage nach unterschiedlichen Rechtsgrundlagen erstellt werden müssen, was zu einem erheblichen Konsolidierungsaufwand führt. Da Gesamtabschlüsse bisher nur in vergleichsweise wenigen Fällen erstellt wurden - vorliegende gesetzliche Regelungen sehen längere Übergangsfristen vor -, wird sich die hier 
liegende Problematik für die Praxis erst künftig in voller Breite stellen, kann also großenteils noch durch legislatorische Maßnahmen aufgefangen werden.

Es dürfte angebracht sein, in der Interpretation der hier angesprochenen Probleme wie auch in Bezug auf die zweckmäßig zu beschreitenden Wege zu ihrer Lösung zu einem möglichst breiten Konsens und zu geeigneten Maßnahmen zu gelangen.

\section{Literaturverzeichnis}

Bauer, Ludwig, Ulrich Kirchmann und Johannes Saß (2009), Mögliche Auswirkungen der Änderungen durch das Bilanzrechtsmodernisierungsgesetz (BilMoG) auf die kommunale Doppik, in: Die Wirtschaftsprüfung 2009, S. 143.

Bieg, Hartmut u. a. (2008), Die Saarbrücker Initiative gegen den Fair Value, in: Der Betrieb 2008, S. 2549.

Bolsenkötter, Heinz, Horst Dau und Eckhard Zuschlag (2004), Gemeindliche Eigenbetriebe und Anstalten - Länderübergreifende Darstellung, 5 Aufl., Stuttgart.

Bolsenkötter, Heinz (Hrsg.) (2007), Die Zukunft des öffentlichen Rechnungswesens - Reformtendenzen und internationale Entwicklungen, Baden-Baden.

Bolsenkötter, Heinz (2008), Modernisierung des Bilanzrechts - Auswirkungen auf öffentliche Unternehmen und Verwaltungen, in: Zeitschrift für öffentliche und gemeinwirtschaftliche Unternehmen, S. 302.

Bundesregierung, Entwurf eines Gesetzes zur Modernisierung des Bilanzrechts (Bilanzrechtsmodernisierungsgesetz - BilMoG) vom 30.07.2008 (mit ergänzenden Materialien). BT-Drs. 16/10067.

Ellerich, Marian (2009), Keine Auswirkungen des BilMoG auf den kommunalen Jahresabschluss? - Zugleich ein Plädoyer gegen allzu bunte Blumensträuße, in: Die Wirtschaftsprüfung, Heft 7 (Editorial).

Ernst, Christoph und Holger Seidler (2009), Gesetz zur Modernisierung des Bilanzrechts nach Verabschiedung durch den Bundestag, in: Betriebs-Berater, S. 766.

Gesetz zur Modernisierung des Bilanzrechts (Bilanzrechtsmodernisierungsgesetz - BilMoG) vom 25. Mai 2009, BGBl. I vom 28. Mai 2009, S. 1102.

Institut der Wirtschaftsprüfer in Deutschland e. V. (2009a), Entwurf IDW Stellungnahme zur Rechnungslegung: Übergangsregelung der Bilanzrechtsmodernisierungsgesetzes (IDW ERS HFA 28), in: IDW Fachnachrichten, S. 344.

Institut der Wirtschaftsprüfer in Deutschland e. V. (2009b), IDW Stellungnahme zur Rechnungslegung: Einzelfragen zur Bilanzierung latenter Steuern nach den Vorschriften des HGB in der Fassung des Bilanzrechtsmodernisierungsgesetzes (IDW ERS HFA 27), in: IDW Fachnachrichten, S. 337.

Land Brandenburg, Verordnung über die Eigenbetriebe der Gemeinden (Eigenbetriebsverordnung - EigV) vom 26. März 2009, GVBl Brandenburg, Teil II, vom 27. April 2009, S. 150.

Marettek, Christian, Andreas Hellenbrand und Peter Detemple (2009), Doppische Kommunal-Haushalte richtig gestalten, Freiburg-Berlin-München.

Meier, Karin (2009), Bilanzierung betrieblicher Versorgungsverpflichtungen nach dem BilMoG, in: BetriebsBerater, S. 998.

Mühlenkamp, Holger und Andreas Glöckner (2009), Rechtsvergleich kommunale Doppik - Eine Synopse und Analyse ausgewählter Themenfelder des neuen, doppischen Haushaltsrechts der Bundesländer, Speyer.

Osar, Peter, Norbert Roß, Dominic Wader und Steffen Drögemüller (2009), Änderungen des Bilanzrechts durch das Bilanzrechtsmodernisierungsgesetz (BilMoG), in: Die Wirtschaftsprüfung, S. 573.

PricewaterhouseCoopers (Hrsg.) (2009), Der reformierte öffentliche Haushalt - Stand nationaler Reformen und internationale Reformtendenzen, Stuttgart.

Rechtsauschuss des Deutschen Bundestags, Beschlussempfehlung und Bericht zu dem Gesetzentwurf der Bundesregierung - Drucksache 16/10067 - (mit Synopse zu den empfohlenen Änderungen) vom 24.03.2009, BT-Drs. $16 / 12407$.

Vogelpoth, Norbert, Michael Poullie und Katharina Voß (2009), Probleme im Zusammenwirken von HGB, BilMoG und kommunaler Rechnungslegung, in: Die Wirtschaftsprüfung, S. 83.

Weigl, Roland, Hans-Georg Weber und Martin Costa (2009), Bilanzierung von Rückstellungen nach dem BilMoG, in: Betriebs-Berater, S. 1062.

Zülch, Henning und Sebastian Hoffmann (2009), Die Modernisierung des deutschen Handelsbilanzrechts durch das BilMoG: Wesentliche Alt- und Neuregelungen im Überblick, in: Der Betrieb, S. 745. 